\title{
Timestamp Error Detection and Estimation for PMU Data based on Linear Correlation between Relative Phase Angle and Frequency
}

\author{
Wenpeng $\mathrm{Yu}$ \\ UTK \\ wyu10@utk.edu \\ (UTK: The University of Tennessee, Knoxville.
}

\author{
Yinfeng Zhao \\ UTK \\ yzhao53@ vols.utk.edu \\ ORNL: Oak Ridge National Laboratory:)
}

\begin{abstract}
Time synchronization is essential to synchrophasor-based applications. However, Timestamp Error (TE) in synchrophasor data can result in application failures. This paper proposes a method for TE detection based on the linear correlation between frequency and relative phase angle. The TE converts the short-term relative phase angle from noise-like signal to one that is linear with the frequency. Pearson Correlation Coefficient (PCC) is applied to measure the linear correlation and then detect the timestamp error. The time error is estimated based on the variation of frequency and relative phase angle. Case studies with actual synchrophasor data demonstrate the effectiveness of TE detection and excellent accuracy of TE estimation.
\end{abstract}

Key words- timestamp error, frequency, relative phase angle, linear correlation, synchrophasor, PMU.

\section{Introduction}

More and more Phasor Measurement Units (PMU) are widely deployed in the power system, and the synchronized measurement enhances the system situational awareness and dynamics observation [1][2]. PMU-based applications rely on the timestamp attached to each measurement to align the data from different PMUs. PMU synchronizes its time with GPS, and normally the accuracy is within 100-nanosecond. However, the time synchronization may be affected by multiple factors, such as poor GPS signal, hardware malfunction, leap second event and GPS time rollover [3][4]. Besides, phasor calculation will use data from a time window of several $60 / 50 \mathrm{~Hz}$ cycles and different vendor may use different approach to assign the calculated value from the beginning to the end of the

This work was supported primarily by the Engineering Research Center Program of the National Science Foundation and the Department of Energy under NSF Award Number EEC-1041877 and the CURENT Industry Partnership Program. time window. This inconsistency will introduce time synchronization errors. Both PPS (Pulse Per Second) signal and serial data of GPS module are needed to form a correct timestamp. The accuracy of PPS can be within a few nanoseconds, but the serial time has a much larger latency, which can be as large as half second. Improperly aligning the serial time with PPS may introduce time error of integer seconds. The inaccuracy of the sampling clock associated with specific PMUs can also introduce time skew issue [5]. The existence of Timestamp Error (TE) in PMU data will adversely influence the performance of PMU-based applications, such as PMU-based protection [6], fault location [7], inter-area oscillation detection [8][9] and event triangulation [10]. Since an increasing number of PMUs are deployed in power systems, it is necessary to detect the timestamp error and estimate the error to resolve the issue.

Different type of timestamp error will yield different signatures. This paper focus on constant timestamp error and a new method is proposed to detect the constant TE based on the linear correlation between frequency and relative phase angle. The short-term relative phase angle, which is mostly determined by the power of millions of loads, is a noise-like signal. However, when constant TE exists, a component that is linear with the frequency will be introduced to the relative phase angle by the time error. Pearson Correlation Coefficient (PCC) [11] is applied to measure the linear correlative, based on which, the timestamp error is detected. The magnitude of the component, which is introduced by $\mathrm{TE}$, is in direct proportion to the time error and frequency. Thus the variation of relative phase angle and frequency are calculated, based on which, the constant time error is estimated. Case study with FNET PMUs demonstrates the effectiveness of the proposed method.

This paper is organized as follows: Section 2 mathematically describes the methodology of proposed TE detection and estimation; Section 3 discussed the detailed algorithm to apply the proposed method; Section 4 presents the experimental verification with 
case studies using actual PMU data from FNET/Grideye. The paper is concluded in Section 5.

\section{Methodology}

\subsection{Timestamp Error Detection}

The real-time phase angle of the complex voltage/current signal changes at the rate of $2 \pi f$, where $\mathrm{f}$ is the frequency of the measured signal. However, the phase angle measurement is the instant angle value at the sampling time [12] and its value is limited within the range of $(-\pi, \pi]$. The phase angle measurement is integral of frequency, and in another word, differential of phase angle is the frequency, as shown in Eq. (1) and (2)

$$
\begin{gathered}
A=2 \pi \int\left(f-f_{N}\right) \cdot d t \\
d A=2 \pi\left(f-f_{N}\right) \cdot d t
\end{gathered}
$$

where $A$ and $f$ are phase angle and frequency, both of which are functions of time $t, f_{N}$ is the nominal frequency, e.g. $50 \mathrm{~Hz}$ or $60 \mathrm{~Hz}$.

Then Eq. (3) is derived from Eq. (2)

$$
A(t+\Delta t)-A(t) \approx 2 \pi\left[f(t)-f_{N}\right] \cdot \Delta t
$$

where $\Delta t$ is a deviation of time.

Supposing the timestamp of synchrophasor data from one PMU has a constant time error $\tau$, Eq. (4) is derived by replacing $\Delta t$ in Eq. (3) with time error $\tau$ :

$$
A(t+\tau) \approx A(t)+2 \pi f(t) \cdot \tau+C
$$

where $C$ is a constant equal to $2 \pi f_{N} \cdot \tau, A(t+\tau)$ is the phase angle with timestamp error, while $A(t)$ is the normal phase angle with accurate time.

Relative phase angle $A_{\text {rel }}(t)$ is defined by Eq. (5), where $A_{r e f}(t)$ is the phase angle of reference PMU. Relative phase angle is commonly used in synchrophasor-data-based applications to detrend the phase angle and reflect the variation of power flow between areas.

$$
A_{\text {rel }}(t)=A(t)-A_{\text {ref }}(t)
$$

By combining Eq. (4) and (5), Eq. (6) is then derived, where $A_{r e l}(t+\tau)$ is the relative phase angle with timestamp error $\tau$.

$$
A_{r e l}(t+\tau) \approx A_{\text {rel }}(t)+2 \pi f(t) \cdot \tau+C
$$

Relative phase angle $A_{\text {rel }}(t)$ is mostly determined by the power flow between areas, which is further determined by the load of millions of end users, whose short term power are unpredictable, making the short term relative phase angle $A_{\text {rel }}(t)$ a noise-like signal, as shown in Fig. 2(b), curve I. However, when the timestamp error $\tau$ is constant, $2 \pi f(t) \cdot \tau$ in the right side of Eq. (6) will be linear with its frequency, which makes the relative phase angle with timestamp error linear with its frequency.

Thus the timestamp error of a PMU can be detected based on the linear correlation between its relative phase angle and frequency. If significant linear correlation is found between the two signals, there is high probability that the PMU has timestamp error issue.

In statistics, the Pearson Correlation Coefficient (PCC) is a measure of the linear correlation between two variables. Thus PCC is applied to quantify the linear correlation between $A_{\text {rel }}$ and $f$. PCC $\rho$ is calculated as Eq. (7), where $\operatorname{cov}($.$) and \sigma$ represent covariance and standard deviation functions respectively.

$$
\rho_{A_{\text {rel }}, f}=\frac{\operatorname{cov}\left(A_{\text {rel }}, f\right)}{\sigma_{A_{\text {rel }}} \sigma_{f}}
$$

Timestamp error is detected when $\rho_{A_{\text {rel }}, f}$ exceeds a threshold $\varepsilon$, where $\varepsilon$ can be selected via simulation study based on historical ambient synchrophasor data with artificial timestamp errors.

\subsection{Timestamp Error Estimation}

From Eq. (6), Eq. (8) can be derived, where $A_{r e l}^{*}$ is the relative phase angle with time error, $\Delta \boldsymbol{X}\left(t_{2}, t_{1}\right)$ denotes the variation of $\boldsymbol{X}$ from time $t_{1}$ to $t_{2}$.

$$
\Delta A_{r e l}^{*}\left(t_{2}, t_{1}\right) \approx \Delta A_{\text {rel }}\left(t_{2}, t_{1}\right)+\Delta f\left(t_{2}, t_{1}\right) \cdot 2 \pi \cdot \tau
$$

Since the normal relative phase angle is a noise-like signal, its short term variation $\Delta A_{\text {rel }}\left(t_{2}, t_{1}\right)$ in Eq. (8) can be ignored. Then Eq. (9) can be derived to estimate the timestamp error.

$$
\tau \approx \frac{\Delta A_{r e l}^{*}\left(t_{2}, t_{1}\right)}{2 \pi \cdot \Delta f\left(t_{2}, t_{1}\right)}
$$

\section{Algorithm}

Online algorithm is developed to detect and estimate the constant timestamp error. The flow chart of the algorithm is shown in Fig.1.

A reference PMU has to be selected to calculate the relative phase angle. Any PMU with good data quality and accurate time can serve as reference. If it is unclear which PMU has accurate time, the system phase angle [12] can be used as reference to achieve the best robustness.

For each time window, the ambient data in 200 seconds is collected, based on which, relative phase angle is calculated and PCC $\rho$ between relative phase angle and frequency is then calculated and compared to the threshold to detect the timestamp error.

To estimate the timestamp error, $t_{1}$ and $t_{2}$ in Eq. (9) are chosen as the time of maximum and minimum frequency in the time window. To improve the estimation accuracy, multiple time windows are 
analyzed, $\Delta A_{r e l}^{*}\left(t_{2}, t_{1}\right)$ and $2 \pi \cdot \Delta f\left(t_{2}, t_{1}\right)$ of each window are collected, Least Squares Method is then applied to obtain a best estimation of timestamp error based on Eq. (9).

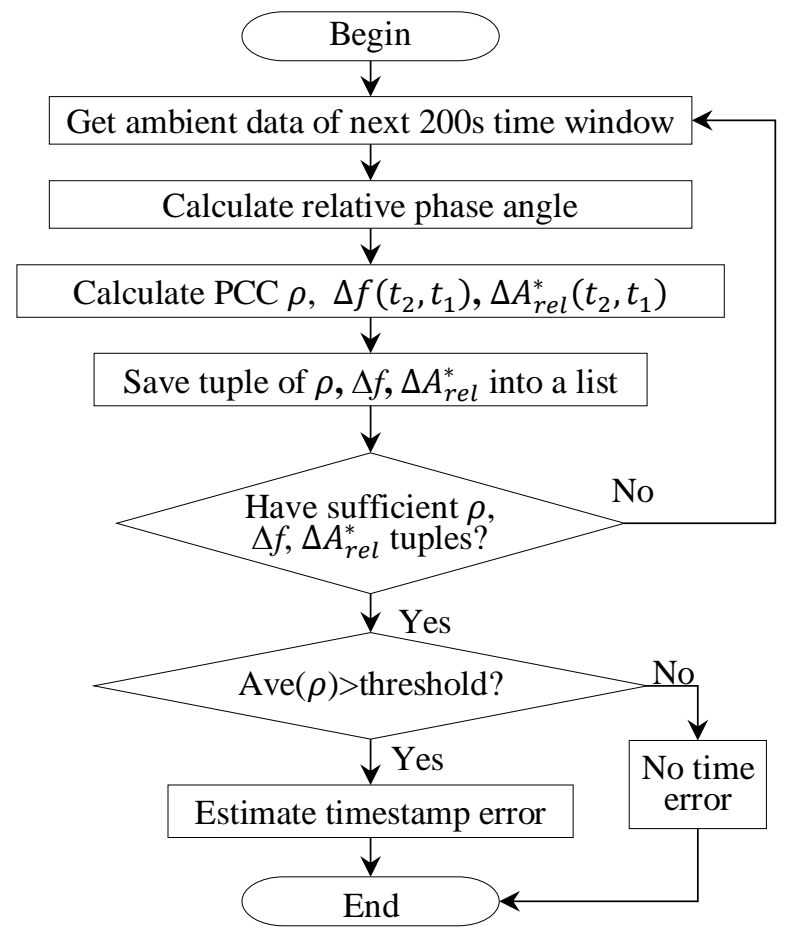

Fig. 1. Flowchart of time error detection and estimation.

\section{Case Study}

To validate the proposed method of constant timestamp error detection and estimation, two cases are studied in this section, and the sensitivity of the detection algorithm and estimation accuracy are discussed too.

FNET/Grideye has deployed hundreds of PMUs in the U.S. Data from FNET is leveraged in the case study.

\subsection{Simulated Timestamp Error and Sensitivity Analysis}

In this case study, a PMU located in Virginia (VA), which has accurate timestamp, is chosen to simulate the timestamp error. To calculate relative phase angle, a PMU located in Tennessee is selected as the reference unit. The ambient data of a 200 -second time window in $05 / 30 / 20198 \mathrm{AM}$ is collected as the raw data. Then 3 simulated measurement series are generated by artificially shifting the time of VA unit by $-0.6 \mathrm{~s},-0.2 \mathrm{~s}$ and 0.3 s respectively.

Frequency of the raw data as well as simulated series are plotted as Fig. 2(a), from which the artificial time error is difficult to observe. Relative phase angle of original and simulated VA unit are calculated and plotted in Fig. 2(b). Curve (I) is the relative phase angle of VA unit, which is a noise-like signal; curve (II), (III) and (IV) are relative phase angle with timestamp shifted by $0.3 \mathrm{~s},-0.2 \mathrm{~s}$ and $-0.6 \mathrm{~s}$. The PCC of the frequency to curve (II)-(IV) are 0.9798, -0.9734 and -0.9850, indicating significant linear correlation between frequency and relative phase angle. With threshold $\varepsilon$ setting to 0.95 , the simulated timestamp error can be detected.

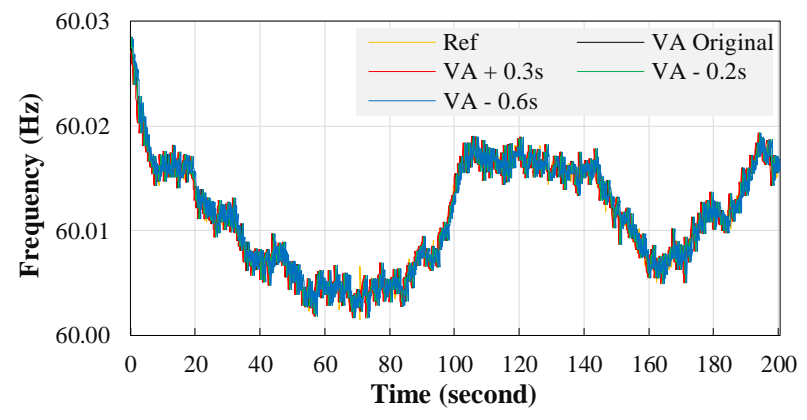

(a) Frequency of reference, original and time shifted VA unit

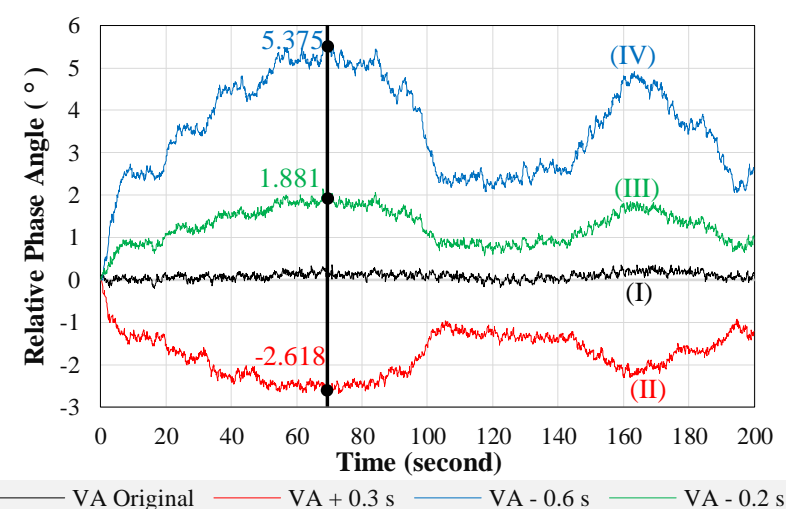

(b) Relative angle of original and time-shifted VA unit

Fig. 2. Simulation of multiple timestamp error.

To estimate the timestamp error, time 0 and 69.8 seconds are chosen as $t_{1}$ and $t_{2}$ in Eq. (9), when frequency reaches its extreme value. The corresponding $\Delta f$ is $-0.0255 \mathrm{~Hz}$, and $\Delta A_{\text {rel }}$ are $-2.618,1.881$ and 5.375 for curve (II)-(IV). According to Eq. (9), the estimated timestamp errors are $0.285 \mathrm{~s},-0.205 \mathrm{~s}$ and $-0.586 \mathrm{~s}$, and corresponding estimation accuracy is $95.6 \%, 97.5 \%$ and 97.6\%, demonstrating excellent estimation effectiveness. 


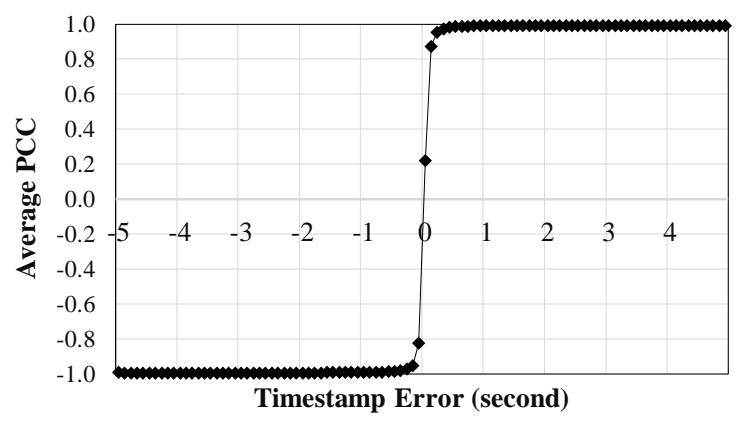

Fig. 3. Sensitivity of PCC with timestamp error.

In order to analyze the sensitivity of the proposed detection method, the timestamp of VA unit is shifted gradually from -5 seconds to 5 seconds with step of $0.1 \mathrm{~s}$ seconds, then the corresponding average PCC of frequency and relative phase angle in 24 hours are calculated and plotted in Fig.3.

When the timestamp error is 0 , the average PCC is around 0.22 , which denotes no linear correlation between frequency and relative phase angle. With \pm 0.1 second timestamp error, the average PCC dramatically increase to 0.875 or -0.822 , indicating high sensitivity of the proposed method for timestamp error detection. With time error larger than \pm 0.2 second, the absolute average PCC is larger than 0.95 .

Based on the sensitivity analysis, the threshold $\varepsilon$ can be set to 0.95 to detect time error larger than 0.2 seconds. To detect time error larger than 0.1 second, $\varepsilon$ can be set to 0.8 .

\subsection{Actual Timestamp Error Case Analysis and Estimation}

FNET/Grideye system has more than 100 PMUs deployed in the Eastern Interconnection (EI) of the U.S. One PMU located in Florida (FL) has a known issue of around 0.9-second timestamp error. Another PMU located in FNET lab for testing has -0.5 second timestamp error. Together with another 18 PMUs, their data in 2019/05/30 are analyzed to detected and estimate the timestamp error. Each time window is 200 seconds, PCC of frequency and relative phase angle, $\Delta A_{r e l}$ and $\Delta f$ in each window are calculated. More than 300 time windows in 24 hours are analyzed.

Frequency and relative phase angle of one 200second time window in 0 A.M. are plotted in Fig. 4 as an example. From the frequency plot, the timestamp error is hardly observed; on the contrary, the plot of relative phase angle of FL unit and FNET Lab unit obviously shows the linear correlation with the frequency, and further indicates timestamp error.

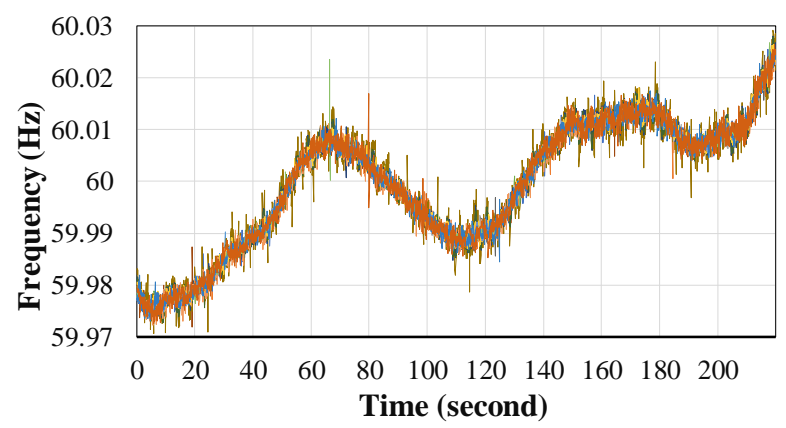

(a) Frequency measurement of 20 EI PMUs

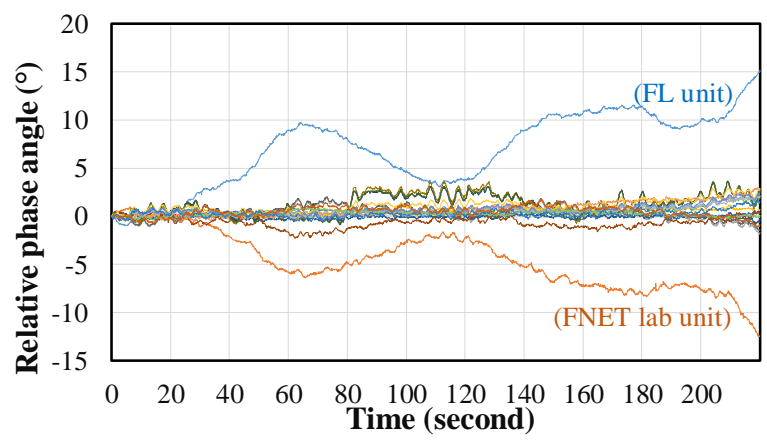

(b) Relative phase angle of 20 EI PMUs

Fig. 4. Frequency and relative angle of EI PMUs.

For each time window, $t_{1}$ and $t_{2}$ in Eq. (9) are chosen as the time of maximum and minimum frequency, then $\Delta A_{\text {rel }}\left(t_{2}, t_{1}\right)$ and $\Delta f\left(t_{2}, t_{1}\right)$ of more than 300 time windows in 24 hours are collected. Least square method is implemented to obtained an optimal estimation of the timestamp error.

$\Delta A_{r e l}$ and $\Delta f$ of FL unit and FNET Lab unit in 24 hours are plotted in Fig. 5. The horizontal axis is $2 \pi \Delta f$, and according to Eq. (9) the slope of the trend line is the estimation of timestamp error.

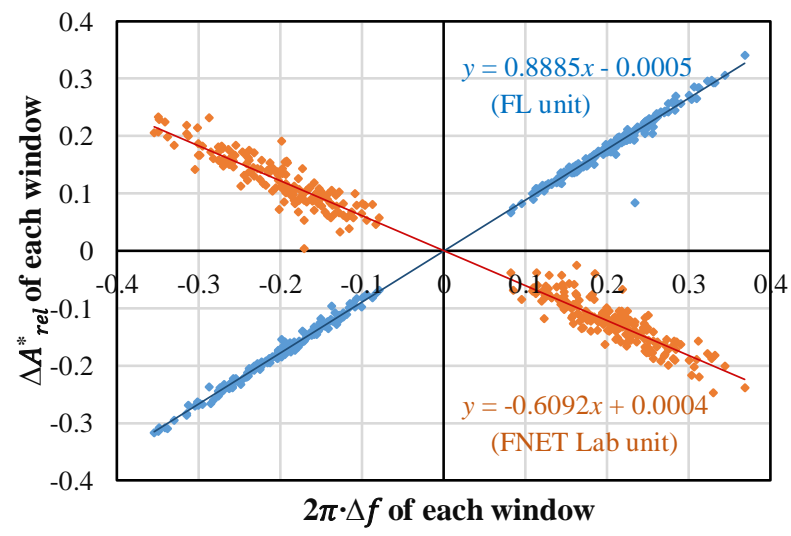

Fig. 5. Scatter of frequency and relative phase angle variation

As shown in Fig.5, the estimated timestamp errors are $0.8885 \mathrm{~s}$ and $-0.6092 \mathrm{~s}$ for FL unit and FNET Lab unit, and the estimation accuracy are $98.72 \%$ and 98.47\% respectively. Comparing with the estimation 
accuracy based on single time window in study case 4.1, the accuracy is improved with multiple time windows.

\section{Conclusion}

A universal methodology is presented in this paper for constant timestamp error detection and estimation in synchrophasor data. The constant timestamp error introduces linear correlation to relative phase angle and frequency, based on which the timestamp error is detected. Then timestamp error is estimated with the variation of frequency and relative phase angle.

The case studies demonstrate the effectiveness of the proposed method, which improves the PMU data quality and corresponding PMU-based applications.

\section{References}

[1] A. G. Phadke and J. Thorp, Synchronized Phasor Measurements and Their Applications. New York, NY, USA: Spring , 2008

[2] Z. Lin et al., "Application of wide area measurement systems to islanding detection of bulk power systems," IEEE Trans. Power Systems, vol. 28, no. 2, pp. 2006-2015, May 2013.

[3] W. Yao et al., "Impact of GPS Signal Loss and Its Mitigation in Power System Synchronized Measurement Devices," IEEE Trans.Smart Grid, vol. 9, no. 2, pp. 1141-1149, March 2018.

[4] Q. F. Zhang and V. M. Venkatasubramanian, "Synchrophasor time skew: Formulation, detection and correction," 2014 North American Power Symposium (NAPS), Pullman, WA, 2014, pp. 1-6.

[5] Q. Zhang, V. Vittal, G. Heydt et. Al, "The time skew problem in PMU measurements", 2012 IEEE Power and Energy Society General Meeting.

[6] V. Centeno, A. G. Phadke, A. Edris, J. Benton, M. Gaudi, and G. Michel, "An adaptive out-of-step relay for power system protection," IEEE Trans. Power Del., vol. 12, no. 1, pp. 61-71, Jan. 1997.

[7] G. Feng and A. Abur, "Fault Location Using Wide-Area Measurements and Sparse Estimation," IEEE Trans. Power Systems, vol. 31, no. 4, pp. 2938-2945, July 2016.

[8] T. Rauhala, A. M. Gole and P. Järventausta, "Detection of Subsynchronous Torsional Oscillation Frequencies Using Phasor Measurement," IEEE Trans. Power Delivery, vol. 31, no. 1, pp. 11-19, Feb. 2016.

[9] T. Jiang et al., "A Novel Dominant Mode Estimation Method for Analyzing Inter-Area Oscillation in China
Southern Power Grid," IEEE Trans. Smart Grid, vol. 7, no. 5, pp. 2549-2560, Sept. 2016.

[10] J. Zhao, L. Zhan and et al, "Measurement accuracy limitation analysis on synchrophasors," IEEE Power \& Energy Society General Meeting, Denver, CO, 2015, pp. 1-5.

[11] https://en.wikipedia.org/wiki/Pearson_correlation_coeffi cient

[12] W. Yu, W. Yao, Y. Liu, "Definition of System Angle Reference for Distribution Level Synchronized Angle Measurement Applications", IEEE Trans. Power Systems, vol. 34, no. 1, pp. 818-820, Jan. 2019. 